\title{
Does Early Kurmanji Speaking Bilingualism Lead to Better Academic Performance? The Role of Working Memory and Reading Performance
}

\author{
Abootaleb Seadatee-Shamir, \\ AliAkbar Soleimanian \\ Bojnord University, Bojnord, Iran
}

\author{
Zeinab Zahmatkesh \\ Azad University of Tabriz, \\ Tabriz, Iran
}

\author{
Hossein Mahdian \\ Azad University of Bojnord, \\ Bojnord, Iran
}

\begin{abstract}
The relationship among WMC (working memory capacity), reading performance and academic achievement is both logically and theoretically undisputable. However, what may not be as obvious is that such capacity and performance, and as a result, achievement, could be higher among ECBL (early childhood bilingual) students. To reaffirm the obvious and explore what may not be so obvious in some quarters, two groups of randomly selected female third graders (200 Farsi speaking and 200 Farsi and Kurmanji speaking) were tested and compared on these characteristics. The measures used were Riding's instrument for measuring WMC and Kormi-Nouri and associates' tests of reading performance. Moreover, academic achievement was defined to be the average of scores obtained by students at their final school exams in math, science and Farsi. Analysis of regression was then performed to determine which construct served as the best predictor of academic achievement in both groups. Based on the results, ECBL girls were superior to their ML (monolingual) counterparts in all three areas. Findings also showed that both WMC and reading performance are strong predictors of school achievement for both groups. However, the memory capacity was the strongest predictor of achievement for both groups. Possible links among bilingualism and better WMC and reading performance are discussed in order to explain the better school achievement by ECBL students.
\end{abstract}

Keywords: WMC (working memory capacity), reading performance, academic achievement, ECBL (early childhood bilinguals), ML (monolingual)

\section{Introduction}

Bilingualism, or even multilingualism, is an aspect of educational reality and common phenomenon for children in many areas of the world. As a result of immigration or ethnic background, many children grow up in the first few years of life learning to speak one language and then learning another language naturally before attending school (Fontroura \& Siegel, 1995). Although it may seem like a simple concept, it is a broad and somewhat vague term which is not simply applied for those who regularly use more than one language in daily life (Girbau \& Schwartz, 2008). However, concerning the literature, bilingualism tends to have different types

Abootaleb Seadatee-Shamir, assistant professor, Counseling and Educational Psychology Department, Bojnord University.

AliAkbar Soleimanian, assistant professor, Counseling and Educational Psychology Department, Bojnord University.

Zeinab Zahmatkesh, Educational Psychology Department, Azad University of Tabriz.

Hossein Mahdian, assistant professor, Educational Psychology Department, Azad University of Bojnord. 
in regard to age. Thus, to find a clear understanding in this regard, it seems necessary to distinguish among these types. According to age-based categorization of Kohnert and Bates (2002), there are two categories of bilingualism, early and late. In regards to the age, early bilingualism includes three types (i.e., infant, childhood and prepuberty). ECBL (early childhood bilingual) which is the main focus of the current study happens after three years old and before school.

Despite of a growing literature on bilingual children, there are few studies that specifically address ECBL in a multi-language community. Consistent with this idea, we wished to investigate the predictive role of WMC (working memory capacity) and reading performance in academic achievement of ECBL and ML (monolingual) students. One possible framework to capture this aim is Baddeley’s (1986; 1996; 2000) multi-component model. Baddeley and Logie (1999) described WMC as a limited central executive system that interacts with a set of two store systems used for temporary storage of different classes of information, which are the speech-based phonological loop and the visual sketchpad. Both of them are in direct contact with the central executive system which is the third component in this model (Baddeley \& Logie, 1999). Although this model was initially conceptualized with three components, Baddeley (2000) added a fourth component, the episodic buffer, to his model which is responsible for binding information across informational domain and memory subsystems into integrated chunks (Alloway, 2009).

A number of studies have examined WMC and other effectively cognitive and educational variables by typically comparing bilingual learners to ML children. However, our particular interest was to investigate reading performance and academic achievement as two fundamental educational variables, together with WMC in ML and ECBL students. In general, results from studies employing different cognitive-based measures of language show striking advantages of bilinguals. The first evidence for superiority of bilinguals in cognitive matters and executive functioning comes from Kohnert, Windsor, and Yim's (2006) studies which suggested that bilinguals could learn instructional and different reading tasks easily and fluently than their ML counterparts. It happens because, mostly, bilinguals have a very flexible and productive command of school achievement and also different ways of cognitive brain working. Moreover, as the age at which a second language is introduced has a dramatic effect on how the brain develops and works, they often acquire a native-like accent. Consequently, it can be the main advantage of ECBL.

Consistent with these results, there are extensive evidence showing bilingualism advantages in executive functioning using tasks based on response conflict, switching and flexibility (Bialystokand \& Shapero, 2005; Carlson \& Meltzoff, 2008; Martin-Rhee \& Bialystok, 2008). Furthermore, other widely accepted studies (Bialystok, 1988; 1997) of bilingualism development suggested that although the basic features of the language acquisition process in both groups are very similar, bilinguals outperform their ML peers in different cognitive tasks. It is mostly because while ML children confront only one form of linguistic input, bilinguals confront two forms and two channels of linguistic input. Consequently, they have higher levels of met linguistic awareness when compared to their ML peers. In addition, they attain relatively high levels of phonological awareness precisely because of the experience with two language systems and the frequent attention to the phototoxic aspects of language.

In contrast to Bialystok, McBride-Chang, and Luk (2005), Bialystok, Craik, and Ryan (2006), Bialystok and Viswanathan (2009) and other researches (e.g., Bochner, 1996; Kessler \& Quinn, 1980, 1987) who used child participants and argued for the cross-language interactivity, Gollan, Montoya, and Werner (2002) argued 
for a cross-language interference and a negative effect of bilingualism (i.e., disadvantages rather than advantages). Gollan et al. (2002) found that adult bilinguals performed worse than adult ML in both letter and semantic fluency tasks. Their arguments were that information in two languages is active simultaneously and bilinguals are unable to turn one language off when the other one is used. By contrast to MLs, there is no need for competition between the two languages and any subsequent interference. Moreover, Bialystok and Feng's (in press) studies demonstrated that bilingual children develop vocabulary more slowly in each language than ML speakers and perform more poorly on measures of language proficiency.

Although some challenges exist in this area, extensive body of previous research accepted the superiority of bilingual as a shared assumption (Leong, Cheng, \& Tan, 2005). In addition, another relatively common assumption in similar studies is that memory is an important predictor of children's reading performances, language skills (Palladino \& Cornoldi, 2004) and school achievement (Swanson \& Jerman, 2007).

\section{Purpose}

Although extensive research suggested that WMC is integrally related to several domains of cognition, including language, comprehension, reading, writing, arithmetic and academic achievement (DeJong, 1998; Gathercole, Alloway, Willis, \& Adams, 2006; Swanson \& Berninger, 1995) and our knowledge relatively, no study has been undertaken to systematically explore whether growth in WMC and reading underlies growth in ECBL and ML's academic achievement. In addition, as the literature concerning ECBL's effects on children's cognitive abilities, especially academic achievement, is not clear cut and still challenging, our particular interest is to explore the interrelation among these three variables in ML and ECBL students.

So, we addressed three questions: First, what is the role of WMC and reading performances in predicting academic achievement of ML and ECBL students to find whether ECBL could show higher ability compared with ML peers? Second, does ECBL appear to have positive or negative consequences for the development of these three constructs? Third, is there a meaningful difference between two groups in all three constructs? It has to be mentioned that the present study has been conducted in Iran that is a multilingual country where different groups of people, such as Persian (Fars), Kormanj, Turks, Kurds, Arabs, etc., live together. Although they all have one official language (Persian) in schools, universities, governmental departments, etc., they use their mother tongue or second language at home and in the street. Both groups are living in Bojnord city (a multilingual speaking area). They use Persian as school language and ECBL students use Kormanji language as a second language.

\section{Materials/Tasks}

\section{Reading Performance Tasks}

Because there is no standardized reading performance test, using factor analysis, five tasks which were loaded of one factor and all clustered to form reading performance component were selected from a battery of standardized reading comprehension tests for Iranian children (Nouri, Moradi, \& Zardkhane, 2006). This task was specifically designed and its psychometric properties ascertained. This test was composed of five separate and timed paper and pencil subtests. Participants were allowed at the range of two to three minutes to complete each subtest (exact time varies for each subtest). Before administration, the participant was presented with an instruction sheet followed by an answer sheet. Instruction sheet is consisting of a series of sample for every subtest. They were instructed to keep trying and do as fast as possible in each subtest within time limitation and 
mark the correct answer in answer sheet. Experimenter is not allowed to help participant during the administration of the task. When time expired for a subtest, participants were instructed to stop working on that subtest and begin the next. At no point, the participants were allowed to go back to work on previous subtests. Experimenter scored correct answers of each subtest independently. The reading performance score was the sum of all correct answers, across all five subtests. Reading performance subtests are as follows.

Word reading. This task is consisting of three lists. Every list includes 40 words which are arranged according to their frequency in three levels (high, medium and low). Two minutes are considered as time limitation for each list. Participants were asked to read as many words as they can and then mark the correct answer in answer sheet. Then, experimenter scored correct answers for each list independently. The maximum score for every list is 40 and for whole subtest is 120 .

Pseudo word reading. This task was administered to assess phonological skills (awareness). In this subtest, participants were supposed to read 40 pseudo words according to a list presented to them from right to left and from up to down. Participants were instructed not to concentrate on meaning of the words but just read with correct pronunciation (one point for every correct answer). Maximum score in this subtest is 30 .

Text comprehension. This subtest consists of three narrative story texts. Two stories are specifically designed for third grade but one story is common for all five grades of elementary students. Story texts were designed according to their interest, needs, cognitive level and their academic curriculum. The number of words in each text is between 320 to 340. There are eight questions for each text and four alternatives for every single question. Maximum score in this subtest is 24. Internal consistency, as measured by Cronbach's alpha, for the three passages was 0.90 , indicating that these passages as a whole and the answers to the comprehension questions were consistent and reliable. The inter-reliabilities for the answers to the 24 questions of these four passages were respectively $0.88,0.92$ and 0.89 .

Phoneme deletion. This subtest consists of 30 words with two or three phonemes. In the first step, experimenter read every word loudly and asked the participant to omit the expected syllable according to the word list and reproduce the remaining word after him. In some words, first syllable and in some others second or last syllable were omitted. The dependent measure was the number of correct answers. Maximum score in this subtest is 30 .

Letter signs. This subtest consists of three letters from 32 alphabets of Persian letters. Each word printed with a bold font on an independent card was presented to participant. Then experimenter read each letter with a high sound and participant was asked to make as much as words, starting with this letter. Produced word is written on answer sheet by experimenter. An important point in this subtest was that every letter has two written form in Persian. So, both forms were presented in a single card and participant is instructed to make word with both forms within time limitation (one minute for every letter and three minute for all). One point is for every correct produced answer. Maximum score in this subtest is 30 .

\section{WMC}

Information processing. The information processing task (Riding, 2000) is a computer-presented assessment showing railway trains comprising carriages of different colors entering the left end of a station into which the whole train disappears from view and then reappears on the right side with some carriage colors changed. Under the control of the subject, the train enters the left side of the station where it is totally obscured from view and then emerges from the right side of the station with one carriage at a time. The 
colors of some of the carriages are different from that on entry. The number of colors used is seven. The task was to indicate one of two marked keys whether or not each carriage color has changed by pressing. This was done as each carriage emerges from the station. Subjects were able to view the train for as long as they wished before they caused it to enter the station. While it was in the station and obscured from view, they had to remember the carriage colors. When they made the train appear out of the station, they had to judge whether there was a carriage color change and respond while retaining information about the remaining carriages that were still obscured. Except in the case when there was only one carriage, both retention and information processing was required and both the amount to be retained and the quantity of processing increased with the length of the train. Thus, the number of carriages correctly identified was taken as an indication of WMC. The length of the trains ranged from one carriage to five carriages, with four presentations of each train length in ascending order of length. There were, thus, a total of carriages to be assessed. Presentation of the carriage colors and changes was pseudo-random so that each presentation was slightly different but very nearly half of the carriages were unchanged in color on each presentation. The score was the percentage of judgments that were correct. The information processing index was individually administered in a computer room with typically 14 pupils per session.

\section{Academic Achievement}

The average scores obtained by students at their final school exams in math, science and Farsi were selected as the student's measure of academic achievement.

\section{Procedure}

A total of 400 third grade children aged between ten and 11 years from seven female state elementary schools in Bojnord, who were rated as having average family socioeconomic status took part in the study. Three classes (nearly 25-30 members) were randomly selected from each school. Then, they were grouped according to their self-reported ML or bilingual status. In terms of socio-economic status, the families of these selected schools were rated as moderate according to students' self-rated report. Before proceeding with task administration, informed consent was gained from the participants. They complete a researcher-made form which was to distinguish among different categories of bilingualism. Then, participants were informed that the project would be conducted in two stages in one session. Each participant was tested individually. The language of experiment was Persian for all ML and ECBL participants. Due to the high population of the study, five fully instructed bilingual (Persian and Kormanji speaking) senior research collaborators (three full time and two part-time) from a local university were employed to administer the tasks. They were given five days' intensive training on the rational of the project, the reason and the design of the groups and individual tasks and specific of the administration before their field work in the schools. These assistants all had experience in working on other projects. During the interactive training sessions, they were also offered considerable advice on the tasks, which were further modified and fine tuned before their administration. This experienced assistance was carefully supervised by the first author of the present study to ensure high fidelity of the field work. At the first step, students' last year scores of final exams in math, science and Farsi were gathered as their academic achievement measure. At the next stage, information processing task was conducted. Then, sub-tests of reading performance task were presented respectively. The tasks were administered in a well illuminated room, furnished with a large desk, a computer and a chair situated in front of the desk. The experimenter sat in a chair 
adjacent to the computer desk with the mouse and keyboard situated in front of him to facilitate operation of the tasks. So, experimenters were present for all tasks and participants completed the tasks individually. Following completion of all tasks, participants were debriefed regarding the purpose of the project. Participants who did not meet the criterion for tasks (multilingual and unrelated bilinguals) were not included in final data analysis.

\section{Results}

The results are presented in one section, which are descriptive statistics, correlations matrix, hierarchical regression and ANOVA (analysis of variance) for all constructs respectively. Descriptive statistics are presented in Table 1.

Table 1

Descriptive Statistics for Working Memory Capacity, Reading Performance and Academic Achievement in ML and ECBL

\begin{tabular}{|c|c|c|c|c|c|c|}
\hline$V A R$ & $L A N$ & $N$ & $M$ & $S D$ & MINI & MAX \\
\hline \multirow[t]{2}{*}{ WMC } & M & 200 & 63.2 & 7.83 & 48 & 81 \\
\hline & B & 200 & 70.1 & 8.72 & 51 & 83 \\
\hline \multirow[t]{2}{*}{ ACA } & $\mathrm{M}$ & 200 & 47.3 & 5.11 & 36 & 58 \\
\hline & B & 200 & 50.9 & 3.77 & 41 & 59 \\
\hline \multirow[t]{2}{*}{ SC } & M & 200 & 15.7 & 1.66 & 12 & 19 \\
\hline & B & 200 & 16.5 & 1.46 & 12 & 19 \\
\hline \multirow[t]{2}{*}{ FA } & $\mathrm{M}$ & 200 & 16.5 & 1.85 & 11 & 20 \\
\hline & B & 200 & 17.2 & 1.62 & 14 & 20 \\
\hline \multirow[t]{2}{*}{ MA } & $\mathrm{M}$ & 200 & 15.1 & 2.37 & 10 & 19 \\
\hline & B & 200 & 17.1 & 1.71 & 12 & 20 \\
\hline \multirow[t]{2}{*}{$\mathrm{RP}$} & $\mathrm{M}$ & 200 & 138.3 & 22.82 & 86 & 196 \\
\hline & B & 200 & 158.6 & 28.33 & 110 & 218 \\
\hline \multirow[t]{2}{*}{ WR } & $\mathrm{M}$ & 200 & 79.1 & 10.77 & 58 & 108 \\
\hline & B & 200 & 84.7 & 13. 17 & 61 & 114 \\
\hline \multirow[t]{2}{*}{ PWR } & $\mathrm{M}$ & 200 & 18.5 & 4.85 & 10 & 28 \\
\hline & B & 200 & 22 & 4.28 & 14 & 30 \\
\hline \multirow[t]{2}{*}{ TC } & $\mathrm{M}$ & 200 & 13.9 & 4.02 & 1 & 24 \\
\hline & B & 200 & 17 & 3.61 & 2 & 24 \\
\hline \multirow[t]{2}{*}{ PD } & $\mathrm{M}$ & 200 & 18.3 & 4.13 & 9 & 28 \\
\hline & B & 200 & 22.7 & 15.86 & 12 & 23 \\
\hline \multirow[t]{2}{*}{ LS } & M & 200 & 8.3 & 2.73 & 2 & 17 \\
\hline & B & 200 & 12.1 & 2.23 & 5 & 19 \\
\hline
\end{tabular}

Notes. VAR, LAN, $N, M, S D, M I N I$ and $M A X$ stand for variance, language, number, mean, standard deviation, minimum and maximum respectively; WMC, ACA, SC, FA, MA, RP, WR, SWR, RC, PD, LS stand for working memory capacity, academic achievement, science, Farsi, mathematic, reading performance, word reading, Pseudo word reading, text comprehension, phoneme deletion and letter sign respectively.

Groups of 400 students are shown in Table 1 . All tests were individually administered and there were no missing data in the data set. Preliminary scanning showed that all variables were practically and normally distributed. Correlations matrix are presented in Table 2. A few aspects of the matrix are worth mentioning here. The five reading performance tasks and academic achievement subcomponent have consistent yet moderate correlations in the range of 0.19 to 0.92 and 0.21 to 0.76 in ML and of 0.19 to 0.92 and 0.17 to 0.76 in ECBL, 
respectively. This is consistent with a previous project conducted with primary school children aged nine to12 (Fontroura \& Siegel, 1995). Second, among all the reading performance tasks, phoneme deletion and letter sign have the highest (0.92) and lowest (0.44) correlation in ML and letter sign and phoneme deletion have the highest (0.92) and lowest (0.17) correlation in ECBL, respectively. Concerning academic achievement measures, Farsi (0.82), math (0.21), math (0.50) and science (0.17) have the highest and lowest correlation coefficient in ML and ECBL, respectively. Last, the reading performance measures have high inter-correlation but moderate correlation coefficient with the WMC and academic achievement measures.

Table 2

Correlation Matrix for Monolingual and Bilingual

\begin{tabular}{|c|c|c|c|c|c|c|c|c|c|c|c|c|c|c|c|c|c|c|c|c|c|c|}
\hline & 1 & & 2 & & 3 & & 4 & & 5 & & 6 & & 7 & & 8 & & 9 & & 10 & & 11 & \\
\hline & M & B & M & B & M & B & M & B & M & B & M & B & M & B & M & B & M & B & M & B & M & B \\
\hline 1 & 1 & 1 & & & & & & & & & & & & & & & & & & & & \\
\hline 2 & $0.68^{* *}$ & $0.75^{* *}$ & 1 & 1 & & & & & & & & & & & & & & & & & & \\
\hline 3 & $0.79^{* *}$ & $0.78^{* *}$ & $0.48^{* * *}$ & $0.54^{* * *}$ & 1 & 1 & & & & & & & & & & & & & & & & \\
\hline 4 & $0.92^{* *}$ & $0.25^{* *}$ & $0.58^{* *}$ & $0.22^{* *}$ & $0.86^{* *}$ & $0.17^{*}$ & 1 & 1 & & & & & & & & & & & & & & \\
\hline 5 & $0.73^{* *}$ & $0.50^{* *}$ & $0.44^{* *}$ & $0.23^{* *}$ & $0.66^{* *}$ & $0.44^{* *}$ & $0.74^{* *}$ & $0.92^{* *}$ & 1 & 1 & & & & & & & & & & & & \\
\hline 6 & $0.35^{* *}$ & $0.34^{* *}$ & $0.27^{* *}$ & $0.22^{* * *}$ & $0.26^{* *}$ & $0.27^{* *}$ & $0.33^{* *}$ & \begin{tabular}{|l|} 
\\
\end{tabular} & $0.28^{* *}$ & $0.46^{* *}$ & 1 & 1 & & & & & & & & & & \\
\hline 7 & $0.28^{* * *}$ & $0.49^{* *}$ & $0.21^{* *}$ & $0.48^{* *}$ & $0.20^{* *}$ & $0.40^{* *}$ & $0.25^{* *}$ & $0.19^{*}$ & $0.21^{* *}$ & $0.33^{* *}$ & $0.82^{* *}$ & $0.45^{* *}$ & 1 & 1 & & & & & & & & \\
\hline 8 & $0.29^{* *}$ & $0.20^{* *}$ & $0.22^{* *}$ & $0.28^{* *}$ & $0.22^{* *}$ & $0.17^{*}$ & $0.28^{* *}$ & $0.18^{*}$ & $0.25^{* *}$ & $0.17^{*}$ & $0.62^{* *}$ & $0.50^{* * *}$ & $0.65^{* *}$ & $0.29^{* *}$ & 1 & 1 & & & & & & \\
\hline 9 & $0.39^{* *}$ & $0.52^{* *}$ & $0.37^{* *}$ & $0.52^{* *}$ & $0.31^{* *}$ & $0.39^{* * *}$ & $0.35^{* *}$ & $0.17^{*}$ & $0.42^{* *}$ & $0.34^{* *}$ & $0.28^{* *}$ & $0.53^{* *}$ & $0.32^{* *}$ & $0.66^{* *}$ & $0.19^{* *}$ & $0.34^{* *}$ & 1 & 1 & & & & \\
\hline 10 & $0.63^{* *}$ & $0.78^{* *}$ & $0.66^{* *}$ & $0.79^{* *}$ & $0.60^{* *}$ & $0.63^{* *}$ & $0.69^{* *}$ & $0.72^{* *}$ & $0.74^{* *}$ & $0.37^{* *}$ & $0.34^{* *}$ & $0.31^{* *}$ & $0.56^{* *}$ & $0.44^{* *}$ & $0.76^{* *}$ & $0.26^{* *}$ & $0.43^{* *}$ & $0.50^{* *}$ & 1 & 1 & & \\
\hline 11 & $0.73^{* *}$ & $0.43^{* *}$ & $0.76^{* *}$ & $0.72^{* *}$ & $0.70^{* *}$ & $0.35^{* *}$ & $0.70^{* *}$ & $0.17^{*}$ & $0.64^{* *}$ & $0.41^{* *}$ & $0.56^{* *}$ & $0.85^{* *}$ & $0.58^{* *}$ & $0.72^{* *}$ & $0.70^{* *}$ & $0.77^{* *}$ & $0.53^{* *}$ & $0.64^{* *}$ & $0.63^{* *}$ & $0.53^{* *}$ & 1 & 1 \\
\hline
\end{tabular}

Notes. $^{*}=(p<0.01)$ and ${ }^{* *}=(p<0.05) ; 1,2,3,4,5,6,7,8,9,10,11$ stand for word reading, pseudo word reading, text comprehension, phoneme deletion, letter sign, science, Farsi, mathematic reading performance, working memory capacity and academic achievement respectively; $\mathrm{M}$ and $\mathrm{B}$ stand for monolingual and bilingual respectively.

Hierarchical regression for prediction of academic achievement in Table 3 shows that in the first step, working memory was entered to regression equation. Results showed that $f_{(84.57)}$ in the level of 0.001 with degree of freedom $(1,401)$ is significant and acquired $R^{2}$ according to this variable is 0.017 . In other word, working memory can predict 0.017 of the variance of academic achievement. In the second step, reading performance was entered to regression equation. Results show that $f_{(72.77)}$ in 0.001 level with degree of freedom $(1,400)$ is significant and acquired $R^{2}$ is 0.026 according to these two variables. In other word, working memory and reading performance can predict 0.026 of the variance of academic achievement. $R^{2} \Delta$ for reading performance is 0.09 . It means that reading performance can predict only 0.09 of academic achievement variance. In the third step, language was entered to regression equation. Regression effect of three variables is $f_{(50.65)}$ in the levels of 0.001 with degree of freedom $(1,399)$ is significant and acquired $R^{2}$ is 0.031 according to these three variables. In other word, working memory and reading performance and language can predict 0.031 of the variance of academic achievement. But, $R^{2} \Delta$ for language is 0.05 .

It means that reading performance can predict only 0.05 of academic achievement variance. This finding shows that working memory, reading performance and language can significantly predict academic achievement respectively. ANOVA which is presented in Table 4 shows that ECBL group was superior to their ML counterparts in all variables except in two of the reading performance tests' sub-variable (phoneme deletion and pseudo word reading). 
Table 3

Hierarchical Regression for Prediction of Academic Achievement

\begin{tabular}{llllllllll}
\hline Variable & $R$ & $R^{2}$ & $R^{2} \Delta$ & $F$ & $D f 1$ & $D f 2$ & Sig. & $\beta$ & $t$ \\
\hline WMC & 0.041 & 0.017 & 0.017 & 84.57 & 1 & 401 & 0.000 & 0.327 & 7.38 \\
Rp & 0.051 & 0.026 & 0.09 & 77.72 & 1 & 400 & 0.000 & 0.301 & 6.75 \\
Lang & 0.052 & 0.031 & 0.05 & 50.65 & 1 & 399 & 0.260 & 0.970 & 2.23 \\
\hline
\end{tabular}

Notes. $R, R^{2}, R^{2} \Delta, D f$, Sig, $\beta$ and $t$ stand for relationship, squared relationship, adjusted relationship, degree of freedom, significance, beta and $t$ test; Lang stands for language.

Table 4

Analysis of Variance for Working Memory Capacity, Reading Performance and Academic Achievement in ML and ECBL

\begin{tabular}{|c|c|c|c|c|c|}
\hline & & SS & $D F$ & MS & $F$ \\
\hline \multirow{3}{*}{ ACA } & BG & $7,663.151$ & 2 & 628.110 & 30.983 \\
\hline & WG & $8,919.370$ & 398 & 20.273 & \\
\hline & $\mathrm{T}$ & $4,583.163$ & 400 & & \\
\hline \multirow{3}{*}{ WMC } & BG & $30,529.958$ & 2 & $2,291.581$ & 33.384 \\
\hline & WG & $39,448.953$ & 398 & 68.642 & \\
\hline & $\mathrm{T}$ & $24,960.330$ & 400 & & \\
\hline \multirow{3}{*}{ RP } & BG & $1,256.219$ & 2 & $1,9724.477$ & 29.870 \\
\hline & WG & $7,663.151$ & 398 & 660.342 & \\
\hline & $\mathrm{T}$ & $8,919.370$ & 400 & & \\
\hline
\end{tabular}

Notes. SS, DF, MS and F stand for sum of square degree of freedom, mean of square and $f$ test in turn; BG, WG and T stand for between group, within group and total respectively.

\section{Discussion}

The purpose of this study was to investigate the role of WMC and reading performance on the prediction of academic achievement in ML and ECBL students. The results suggested a moderate link between these variables in both groups. As such, these data provide support for previous claims that WMC is an essential aspect of reading performance and academic achievement in bilingual student (Bull, Espy, \& Wiebe, 2008; Fontoura \& Siegel, 1995; Gathercole et al., 2006; Gropper \& Tannock, 2009; Moehara \& Saitos, 2007). In addition, these findings have implications for classic cognitive ability studies linking reading performance to academic achievement (Andersson \& Lyxell, 2007; T. P. Alloway \& R. G. Alloway, 2010; Alloway, Gathercole, Kirkwood, \& Elliott, 2009; Gathercole et al., 2006; Swanson \& O’Connor, 2009; Swanson \& Jerman, 2007). The current results suggest that the tasks used to measure WMC in those classic studies may have tapped into reading performance to some extent.

The results reported here, as well as prior empirical reports (Seigneuric, Ehrlich, Oakhill, \& Yuill, 2000; Thompson \& Gathercole, 2006; Van der Leij, Bekebrede, \& Kotterink, 2010), lead to the speculation that WMC is the basis of reading performance and academic achievement in ML and ECBL groups. This may sound like a bold statement, because at least two leading researchers on the topic of information processing approaches to WMC and reading performance have made similar claims. Furthermore, the results suggest that ECBL children had significantly higher scores on these three construct than ML counterparts. It means that 
WMC helps ECBL, decodes unfamiliar words, guesses at the meaning of words from context and comprehends complicated text better than ML peers. Also, ECBL with relatively high WMC are better able to hold initial sounds in mind while finishing the decoding of an unfamiliar word and they can process phonemes, letters, ambiguous and pseudo words and sentences more efficiently (Miyake, Just, \& Carpenter, 1994) and are better at guessing the meanings of unfamiliar words based on context (Daneman \& Green, 1986). Generally, WMC has been found to correlate highly with reading performance in ML and bilinguals (Engle, Cantor, \& Carullo, 1992). If a reader has a high capacity for language-based or verbal WMC, then comprehension processes (e.g., word encoding, lexical access, phonemes and letters process, syntactic and semantic analysis) are less of a strain on the limited capacity system (Miyake et al., 1994).

Moreover, findings of the present study provide support for the speculation that bilingual children had significantly higher scores on WMC, reading performance and academic achievement than ML peers (Girbau \& Schwartz, 2008; Fontoura \& Siegel, 1995; Ferrari \& Palladino, 2007; Carlson \& Meltzoff, 2008). The finding concerning ECBL students reveals that they have the capacity to develop creative meaning, association and thinking more naturally than MLs peers. Consequently, they are outperforming in math, science and Farsi comparing with their ML counterparts. Additionally, ECBL seems to have superior WMC and more resources available for concurrent integration and comprehension and they do acquire their dominant language (or both their languages if neither is dominant) to an ability equal to that of ML peers. But, how well an ECBL develops its second language can vary from a child who only knows a few phrases and some very basic vocabulary. To a child, he/she listens and understands, but cannot or perhaps will not speak to a balanced bilingual child (who communicates in both languages) with the same command as their ML peers in both languages.

However, science ECBL students are exposed to more than one language, they can relatively experience the richness of another language better than ML peers. As a result, their cognitive abilities, especially WMC, are rather improved and they will be capable of learning school courses, such as science, Farsi and math more easily and properly than ML counterparts. However, ECBL skills in learning vary from a course like science (in which both groups had the highest performance) to a course like math (in which both groups had the lowest score). This finding is consistent with the current literature suggesting that bilingualism plays an important part in accounting for individual differences in reading performance and academic achievement (Swanson \& O'Connor, 2009). However, the results also indicate that WMC plays a critical role in moderating individual differences in these two variables. In fact, WMC, language skills and reading performance were found to be statistically significant in predicting academic achievement (Saiegh-Haddad \& Geva, 2008).

Still, further findings of the present study in line with studies suggested that bilingual children not only better appreciate what human versus is and what culture is, but they are also more inclined to have a deeper appreciation of language. They understand at an early age that there is more than one way to label or discuss something. They understand that different labels for the same object or idea in different languages can have different connotations. They are more likely to see the creative possibilities of language and explore their own linguistic creativities (Saiegh-Haddad \& Geva, 2010; Martin-Rhee \& Bialystok, 2008; Kohnert \& Bates, 2002). Consequently, they have a better performance in text comprehension than other observed variables of reading performance. In addition, both of them have a better performance in text comprehension than that in other observed variables of reading performance. The present findings also converge on the view that language plays an effective role in cognitive development. Language is a crucial tool in forming cognition, which enables a child to organize abstract thoughts. Segalowitz (1997) suggested that the internalization of two languages rather 
than one results in a more complex and better equipped cognition enables the child to alternate between the two linguistic codes (Gathercole, Willis, Emslie, \& Baddeley, 1992). Furthermore, Kohnert et al. (2006) asserted that, in addition to stretching their memory and minds intellectually, learning two languages allows bilingual persons to develop their instructional and cultural understanding of people beyond them. Therefore, this capability leads ECBL students to have better syntactic, semantic, linguistic and finally school achievement than ML peers.

Our results provide further support for the proposal in the bilingualism literature that being able to step into another culture through its language is like being able to live a second life. Although some ECBL children do not have a lot of exposure to the culture of their second language, the language itself conveys much of the culture to them. Further, even if, ECBL children are not learning the language academically, but they can attain grammatical and semantic language skills orally through communication in their houses or communities. In this case, they are potentially capable of experience more cognitive exercise to develop their cognitive abilities. Moreover, learning two languages simultaneously helps them to extend the storage of the different vowels, sounds and letters, and benefits a rich cognitive and linguistic storage in their memory.

In this case, they can improve their knowledge in different educational areas especially at school, and as a result, attain a better academic achievement than ML peers. Another important issue concerning the nature of bilingualism consistent with the finding of this study is that ECBL children have some experience seeing how different cultures cause different people to interpret completely differently the exact same circumstance. Seeing two different cultures internally helps bilingual children realize that much of what is considered universal human behavior within a culture may be unique to that culture. This awareness and understanding of differences between different properties of two languages prepare children to produce better judgment in regard to instructional position (Swanson, Sez, Gerber, \& Leafstedt, 2004). Additional evidence for the effective role of working memory, rather than reading performance, in the prediction of academic achievement in both ML and ECBL group comes from T. P. Alloway and R. G. Alloway (2010). They suggested valuable implications of working memory for education. Consistent with the finding of the present study, they believed that working memory and reading skills have a strong role in attaining academic achievement. So, they believed that in most educational contexts, especially in the classroom, students have to rely on working memory and reading skills to perform a range of activities. Poor working memory and reading skill lead to failures in simple tasks, such as remembering classroom instructions to more complex activities involving storage and processing of information and keeping track of progress in difficult tasks. In sum, the crucial finding we wish to emphasize is that ECBL children with relatively high WMC and reading performance are more successful in academic achievement than ML peers. Although, there are some little probable disadvantages of ECBL, which play an important role in attaining academic achievement and other cognitive variables, but this study highlights the importance of acquisition of early language skills in two (or more) language speaking families.

\section{Shortcomings}

To the best of our knowledge, our study with a large sample of 400 Iranian children might be among the first to investigate the relative contribution of some cognitive and linguistic skills of these children. We acknowledged some shortcomings in the study. First, the study was confined to ML and ECBL Iranians children in Bojnord between the ages of 12 and 13 in grade five and was not able to assess other grades and also developmental paths and possible changes in the children's performances of the different tasks and their 
interactions. Second, as working memory does, a system comprised separable components, multiple tasks and assessments over time is needed to examine alternative theoretical accounts of WMC (Alloway, Pickering, \& Gathercole, 2006). Third, path analysis with its composite measurement model, describes relations of dependency among the latent constructs and no claim is made to causality. Path model with its acceptable "good" fit and the prediction of Persian text reading from the different path are attempts at answering some research questions and should be interpreted as approximations of reality. Fourth, this study could not investigate the cause-and-effect relationship of bilingualism and the answer to this question is not still obvious. Does bilingualism come first and cause cognitive benefits, do cognitive abilities enhance language learning, or do cognitive development and language learning work hand in hand?

We would, however, also like to suggest that in support of the literature on English reading performance, WMC had a strong effect on Persian text comprehension and, in fact, had the largest joint or unique effects on overall text comprehension (Nation, Adams, Bowyer-Crane, \& Snowling, 1999; Perfetti \& Liu, 2005). The finding of this study extends our understanding of a novel domain of bilingualism in the area of cognitive and school achievement. However, as it is among the first to explore this type of bilingualism, it has some more limitations. Moreover, general language mechanisms underlying reading performance, WMC and academic achievement should be further investigated to clarify this interrelation in ECBL children.

\section{References}

Alloway, T. P. (2009). Working memory, but not IQ predicts subsequent learning in children with learning difficulties. European Journal of Psychological Assessment, 25, 92-98.

Alloway, T. P., \& Alloway, R. G. (2010). Investigating the predictive roles of working memory and IQ in academic attainment. Journal of Experimental Child Psychology, 106, 20-29.

Alloway, T. P., Pickering, S. J., \& Gathercole, S. E. (2006). Verbal and visuospatial short-term and working memory in children: Are they separable? Journal of Child Development, 18, 1698-1716.

Alloway, T. P., Gathercole, S. E., Kirkwood, H., \& Elliott, J. (2009). The working memory rating scale: A classroom-based behavioral assessment of working memory. Learning and Individual Differences, 19, 242-245.

Andersson, U., \& Lyxell, B. (2007). Working memory deficit in children with mathematical difficulties: A general or specific deficit? Journal of Experimental Child Psychology, 96, 197-228.

Baddeley, A. (1996). Exploring the central executive. The Quarterly Journal of Experimental Psychology, 49, 5-28.

Baddeley, A. (2000). The episodic buffer: A new component of working memory? Trends in Cognitive Sciences, 4, 417-423.

Baddeley, A., \& Logie, R. H. (1999). Working memory: The multiple-component model. In A. Miyake, \& P. Shah (Eds.), Models of working memory (pp. 28-61). New York: Cambridge University Press.

Bialystok, E. (1988). Levels of bilingualism and levels of linguistic awareness. Developmental Psychology, 24, 560-567.

Bialystok, E. (1997). Effects of bilingualism and biliteracy on children’s emerging concepts of print. Developmental Psychology, 33, 429-440.

Bialystok, E., \& Feng, X. (n. d.). Language proficiency and its implications for monolingual and bilingual children. Guildford Press (in press).

Bialystokand, E., \& Shapero, D. (2005). Ambiguous benefits: The effect of bilingualism on reversing ambiguous figures. Developmental Science, 595-604.

Bialystok, E., \& Viswanathan, M. (2009). Components of executive control with advantages for bilingual children in two cultures. Journal of Cognition, 112, 494-500.

Bialystok, E., McBride-Chang, C., \& Luk, G. (2005). Bilingualism, language proficiency, and learning to read in two writing systems. Journal of Educational Psychology, 97, 580-590.

Bialystok, E., Craik, F. I., \& Ryan, J. (2006). Executive control in a modified anti-saccade task: Effects of aging and bilingualism. Journal of Experimental Psychology: Learning, Memory, and Cognition, 32, 1341-1354.

Bochner, S. (1996). The learning strategies of bilingual versus monolingual students. British Journal of Educational Psychology, 66, 86-93. 
Bull, R., Espy, K. A., \& Wiebe, S. (2008). Short-term memory, working memory and executive functioning: Longitudinal predictors of mathematics achievement at age 7. Developmental Neuropsychology, 33, 205-228.

Carlson, S. M., \& Meltzoff, A. N. (2008). Bilingual experience and executive functioning in young children. Developmental Science, 11, 282-298.

Daneman, M., \& Green, I. (1986). Individual differences in comprehending and producing words in context. Journal of Memory and Language, 25, 1-18.

DeJong, P. F. (1998). Working memory deficits of reading disabled children. Journal of Experimental Child Psychology, 70, 75-96.

Engle, R. W., Cantor, J., \& Carullo, J. J. (1992). Individual differences in working memory and comprehension: A test of four hypotheses. Journal of Experimental Psychology: Learning, Memory, and Cognition, 18, 972-992.

Engle, R. W., Tuholski, S. W., Laughlin, J. E., \& Conway, A. R. A. (1999). Working memory, short-term memory, and general fluid intelligence: A latent-variable approach. Journal of Experimental Psychology: General, 128, 309-331.

Ferrari, M., \& Palladino, P. (2007). Foreign language learning difficulties in Italian children. Journal of Learning Disabilities, 40, 256-269.

Fontroura, H., \& Siegel, L. S. (1995). Reading, syntactic and working memory skills of bilingual Portuguese-English Canadian children. Reading and Writing, 7, 139-153.

Gathercole, S. E., Willis, C. S., Emslie, H., \& Baddeley, A. D. (1992). Phonological memory and vocabulary development during the early school years: A longitudinal study. Developmental Psychology, 28, 887-898.

Gathercole, S. E., Alloway, T. P., Willis, C., \& Adams, A. M. (2006). Working memory in children with reading disabilities. Journal of Experimental Child Psychology, 93, 265-281.

Girbau, D., \& Schwartz, R. G. (2008). Phonological working memory in Spanish-English bilingual children with and without specific language impairment. Journal of Communication Disorders, 41, 124-145.

Gollan, T. H., Montoya, R. I., \& Werner, G. A. (2002). Semantic and letter fluency in Spanish-English bilinguals. Neuropsychology, 16, 562-576.

Gropper, R. J., \& Tannock, R. (2009). A pilot study of working memory and academic achievement in college students with ADHD. Journal of Attention Disorders, 12, 574-581.

Kessler, C., \& Quinn, M. (1980). Positive effects of bilingualism on science problem solving abilities. Oxford: Oxford University Press.

Kessler, C., \& Quinn, M. (1987). ESL and science learning. In J. Crandall (Ed.), ESL through content-area instruction (pp. 55-87). Englewood Cliffs, N. J.: Prentice Hall Regents.

Kohnert, K. J., \& Bates, E. (2002). Balancing bilinguals II: Lexical comprehension and cognitive processing in children learning Spanish and English. Journal of Speech, Language, and Hearing Research, 45(2), 347-359.

Kohnert, K. J., Windsor, J., \& Yim, D. (2006). Do language-based processing tasks separate children with language impairment from typical bilinguals? Learning Disabilities Research and Practice, 21, 19-29.

Leong, C. K., Cheng, P. W., \& Tan, L. H. (2005). The role of sensitivity to rhymes, phonemes and tones in reading English and Chinese pseudo words. Reading and Writing, 18, 1-26.

Martin-Rhee, M. M., \& Bialystok, E. (2008). The development of two types of inhibitory control in monolingual and bilingual children. Bilingualism: Language and Cognition, 11, 81-93.

Miyake, A., Just, M. A., \& Carpenter, P. A. (1994). Working memory constraints on the resolution of lexical ambiguity: Maintaining multiple interpretations in neutral contexts. Journal of Memory and Language, 33, 175-202.

Moehara, Y., \& Saitos. (2007). The relationship between processing and storage in working memory span: Not two sides of the same coin. Journal of Memory and Language, 13, 212-228.

Nation, K., Adams, J. W., Bowyer-Crane, C. A., \& Snowling, M. J. (1999). Working memory deficits in poor comprehenders reflect underlying language impairments. Journal of Experimental Child Psychology, 73, 139-158.

Nouri, R. K., Moradi, A. R., \& Zardkhane, S. A. (2006). Investigating reading performance status and dyslexia in monolingual (Tehran students) and bilingual (Tabriz and Sannandaj students) elementary school students. Research Center for Education Strategy and Curriculum Development of Iran's Ministry of Education. (in Persian)

Palladino, P., \& Cornoldi, C. (2004). Working memory performance of Italian students with foreign language learning difficulties. Learning and Individual Divergences, 14, 137-151.

Perfetti, C. A., \& Liu, Y. (2005). Orthography to phonology and meaning comparisons across and within writing systems. Reading and Writing: An Interdisciplinary Journal, 18, 193- 210. 
Riding, R. (2000). Information processing index. Birmingham: Learning and Training Technology.

Saiegh-Haddad, E., \& Geva, E. (2008). Morphological awareness, phonological awareness, and reading in English-Arabic bilingual children. Reading and Writing, 21, 481-504.

Saiegh-Haddad, E., \& Geva, E. (2010). Acquiring reading in two languages: An introduction to the special issue. Reading and Writing, 23, 263-267.

Segalowitz, N. (1997). Individual differences in second language acquisition. In A. M. B. de Groot, \& J. F. Kroll (Eds.), Tutorials in bilingualism: Psycholinguistic perspectives (pp. 85-112). Mahwah, N. J.: Lawrence Erlbaum.

Seigneuric, A., Ehrlich, M. F., Oakhill, J., \& Yuill, N. (2000). Working memory resources and children’s reading comprehension. Reading and Writing, 13, 81-103.

Swanson, H. L., \& Berninger, V. (1995). The role of working memory in skilled and less skilled readers' comprehension. Journal of Intelligence, 21, 83-108.

Swanson, H. L., \& Jerman, O. (2007). The influence of working memory on reading growth in subgroups of children with learning disabilities. Journal of Experimental Child Psychology, 96, 249-283.

Swanson, H. L., \& O’Connor, R. (2009). The role of working memory and fluency practice on the reading comprehension of students who are dysfluent readers. Journal of Learning Disabilities, 42, 548-575.

Swanson, H. L., Sez, L., Gerber, M., \& Leafstedt, J. (2004). Literacy and cognitive functioning in bilingual and non-bilingual children at or not at risk for reading disabilities. Journal of Educational Psychology, 96, 3-18.

Thompson, H., \& Gathercole, S. (2006). Executive functions and achievements in school: Shifting, updating, inhibition, and working memory. Quarterly Journal of Experimental Psychology A, 20, 745-759.

Van der Leij, A., Bekebrede, J., \& Kotterink, M. (2010). Acquiring reading and vocabulary in Dutch and English: The effect of concurrent instruction. Reading and Writing, 23, 415-434. 